\title{
Taxonomic study of some medicinally important species of Swertia L. (Gentianaceae) in Nepal
}

\author{
Dilli Prasad Rijal
}

Central Department of Botany, Tribhuvan University, Kirtipur, Kathmandu, Nepal; email: dilliprijal@gmail.com

\begin{abstract}
Among 100 species of Swertia reported from the world, Nepal harbors 30 species along with four varieties. In Nepal, species of Swertia are highly exploited as raw materials for different traditional medicines. Among them, S. chirayita, a vulnerable species, has been considered to be superior in medicine and trade. One of the main issues in its trade is adulteration with other species which are considered to be inferior in medicinal quality. Species which are mixed with S. chirayita are S. angustifolia, S. ciliata, S. dilatata, S. paniculata, S. racemosa, etc. There is a need for the selection of most important morphological characteristics for instant identification of different species of Swertia to check illegal trade as well as adulteration. This paper aims to provide the most important identifying characters of eight species of Swertia traded from Nepal on the basis of their morphological studies. Color of the petal, number of floral parts (tetramerous vs. pentamerous) and number of glands in petal are the key characters for the identification of the species.
\end{abstract}

Key-words: adulteration, medicinal plant, morphological characters.

\section{Introduction}

Gentianaceae is a family of 84 genera and about 970 species in the world (Judd et al. 1999). Members of the family are widely distributed, but are most diverse in temperate and subtropical regions and in the montane tropics. Clarke (1885) divided Gentianaceae into three tribes: Exaceae, Chironeae and Swertieae. Cronquist (1988) treated the family under order Gentianales of subclass Asteridae and class Magnoliopsida. Judd et al. (1999) treated the family under order Gentianales and clade Core asterids.

Swertia is named in honor of Emanuel Sweert (rarely spelled Swert, 1552-1612), a Dutch gardener. Clarke (1885) divided Swertia into three subgenera: Ophelia, Euswertia and Poephila. A total of 29 species of Swertia have been reported from Nepal (Press et al., 2000; DPR, 2001). However, the number reached to 30 as Chassot (2003) recently reported Swertia barunensis from east Nepal.

Nepalese species of Swertia are distributed from east to west and from tropical to alpine zone. Distribution ranges from $600 \mathrm{~m}$ asl (S. angustifolia) to $5600 \mathrm{~m}$ asl (S. petiolata). Certain species, like $S$. angustifolia, S. chirayita and S. multicaulis are highly exploited as raw materials for different medicines. However, $S$. chirayita plays dominant role in trade and is considered to be superior in medicinal quality. Due to high commercial demand, S. chirayita has vulnerable status in Nepal and India. One of the main issues in its trade is adulteration with other low-value species considered to be inferior in medicinal quality (Ghimire et al. 2008). Swertia nervosa is the main substitute of S. chirayita in trade (Pant and Bimb 2005). Other species which are generally mixed with $S$. chirayita are $S$. angustifolia, S. ciliata, S. dilatata, S. paniculata, S. racemosa, etc. (Bhattarai and Acharya 1998; Ghimire et al. 2008). Sometimes adulteration occurs due to misidentification of the true species.
Taxonomically Swertia is a difficult genus. Morphological characters are the main basis for the identification and delimitation of taxa within Swertia. Most importantly there is a need for the selection of pertinent morphological characteristics for instant identification of different species of Swertia involved in trade which will help to check illegal trade as well as adulteration. This paper aims to provide the most important identifying characters of eight species of Swertia traded from Nepal.

\section{Materials and Methods}

The present study was based on the herbarium specimens deposited at Tribhuvan University Central Herbarium (TUCH) and some specimens collected by the author. The vegetative and reproductive parts of the specimens were examined. Identity of the species was confirmed based on the specimens deposited at National Herbarium and Plant Laboratory (KATH), and following standard literature (Zheng-yi and Raven 1995; Grierson and Long 1999).

\section{Results}

\section{TAXONOMIC TREATMENT}

\section{Swertia L.}

Members of the genus are annual, biennial or perennial herb. Roots fibrous or woody. Stems absent, scapiform, or well developed, ascending or erect, terete, striate or angled or winged, simple or branched. Leaves opposite, rarely alternate or whorled or rosulate, sessile or petiolate, margin entire. Inflorescence cymose, usually grouped into simple or paniculate thyrses, rarely strictly dichoto- 
mous, sometimes reduced to single flowers and inflores-cences raceme- like or flowers solitary and terminal. Flowers pedicellate, 4or 5-merous. Bracts leaf-like, opposite, sessile. Calyx and corolla rotate, lobed to base, tubes less than $3 \mathrm{~mm}$. Nectaries 1 or 2 per corolla lobe, naked or covered by a scale or flaps, glabrous, fringed or fimbriate. Stamens as many as corolla lobes, attached at base of corolla lobe sinuses, sometimes surrounded by long hairs. Ovary 1-celled. Style short to elongate. Stigma bilobed. Fruit a capsule, enclosed by persistent calyx and corolla, ovoid or flattened, dehiscing into 2 valves, few to many seeded. Seeds small.

\section{KEY TO THE SPECIES}

1a. Flowers 4-merous

2a. Glands 2 per corolla. 2. S. chirayita

2b. Gland 1 per corolla

3a. Corolla white or pale yellow with brown spots.. 1. S. angustifolia 3b. Corolla pale yellow-green or whitish with purple nerves

6. S. nervosa

1b. Flowers 5-merous

4a. Glands 2 per corolla, plant non-ciliated ............... 4.S. cuneata

4b. Gland 1 per corolla, cilia in leaf margin or sepal or petal

5a. Filament dilated up to the half, 2 greenish spots above each gland in each petal 5.S. dilatata

5b. Filament not dilated, greenish spots absent

6a. Glands linear, covered by fimbriated flap, cilia present in sepal and petal .......................................... 8.S. racemosa

6b. Glands horse-shoe-shaped, covered by non-fimbriated flap, cilia absent in sepal and petal

7a.Corolla reflexed at anthesis, purple band or spots above each gland, no flap and fimbria on gland ....

3.S. ciliata

7b.Corolla not reflexed, 2 blackish spots above each gland, yellow non-fimbriated flap on gland 7.S. paniculata

1. Swertia angustifolia D. Don, Prodr. Fl. Nepal.: 127 (1825). Clarke in Fl. Br. India 4 (10): 125 (1883). Chater in Enum. Fl. Pl. Nepal 3: 96 (1982). Malla et al., Fl. Kath. Valley: 483 (1986). Tingnung \& Pringle in Fl. China 16: 116 (1995). Aitken in Fl. Bhutan 2 (2): 626 (1999). Press et al., Ann. Check. Fl. Pl. Nepal: 117(2000).

Ophelia angustifolia (Buch.-Ham. ex D. Don) G. Don, Gen. Syst. 4: 178 (1837).

Chiraito

Annual herb, 20-80 cm. Stem erect, hollow, quadrangular, glabrous. Leaves subsessile, leaf blade linear to narrow lanceolate, 2-5.5 $\times 0.2-1.7 \mathrm{~cm}$, base attenuate, margin entire, apex acute, veins 1-3. Inflorescence many flowered panicles. Pedicel 0.6-2.3 cm. Bracts linear or lanceolate, $2.3-1.0 \mathrm{~cm} \times 1.0-2.0 \mathrm{~mm}$. Flowers 4-merous. Calyx green, tube $0.5 \mathrm{~mm}$, lobes linear or lanceolate, $0.8-1.4 \mathrm{~cm} \times 1$ $2 \mathrm{~mm}$, 3-nerved. Corolla white or pale yellow, with brown spots, tube $1 \mathrm{~mm}$, lobes elliptic or ovate, 5-7 × 2-4 mm, gland 1, pocketshaped, orbicular scale with short fimbriae. Stamens 4 , filaments $2.5 \mathrm{~mm}$, anther $1 \mathrm{~mm}$. Carpel 1.5-7 mm. Capsule $0.5 \mathrm{~mm}$. Fl. \& Fr. Aug.-Nov. (Fig. 1).
Distribution: Nepal (WCE, 235-2600 m), Himalaya (Kashmir to Bhutan), N. India, Myanmar, S. China.

Specimens examined: Sindhuli: Napani, 1240 m, 27 Nov. 1988, N.P. Manandhar 12637 (KATH). Ramechhap: Kangba gaon, 1850 m, 13 Aug. 1977, K.R. Rajbhandary and B. Roy 2245 (KATH). Kathmandu: Putalidanda,1800 m, 7 Jan. 1975, Joshi et al. 75/134 (KATH); Chobhar, 27 Apr. 2003.,R. Upreti 2 (TUCH); Chobhar, 10 Oct. 2001, B. Raskoti 10 (TUCH). Rasuwa: Syabru bensi, 1450 m, 5 Sept. 1971, J.F. Dobremez 961 (KATH). Dhading: Todaki, 1150 m, 8 Nov. 1989, N.P. Manandhar 13172 (KATH). Chitwan: Tiger Top, 300 m, 12 Dec. 1975, D.P. Joshi et al. 75/ 3498 (KATH). Myagdi: Dana, 1470 m, 30 Aug. 1983, N.P. Manandhar 10282 (KATH); Tripling, 1050 m, 1 Oct. 1985, D.P. Joshi et al. Gulmi: Kudapani, 1 June 2003, K.C. Bishnumaya 62 (TUCH). Palpa: Chilaune danda, 26 Jestha 2058, G. Shreshtha 69 (TUCH). Syangja: Bayar Ghari, 10 June 2001, Do35 (TUCH). Kanchanpur: Suklaphanta, 235 m, 28 March 1984, P. Pradhan and R.K. Uprety 978 (KATH). Kalikot: Takula, 1600 m, 14 Aug. 1981, N.P. Manandhar and D.P. Joshi 8263 (KATH). Doti: JhigadanaChoyagadru, 2500 m, N.K. Bhattarai 90/762 (KATH). Dailekh: Chhanna, 2000 m, N.P. Manandhar and D.P. Joshi 8227 (KATH). Rolpa: Budhogam, 1313 m, 5 Sept. 1982, N.P. Manandhar and N.K. Bhattarai 8809 (KATH).

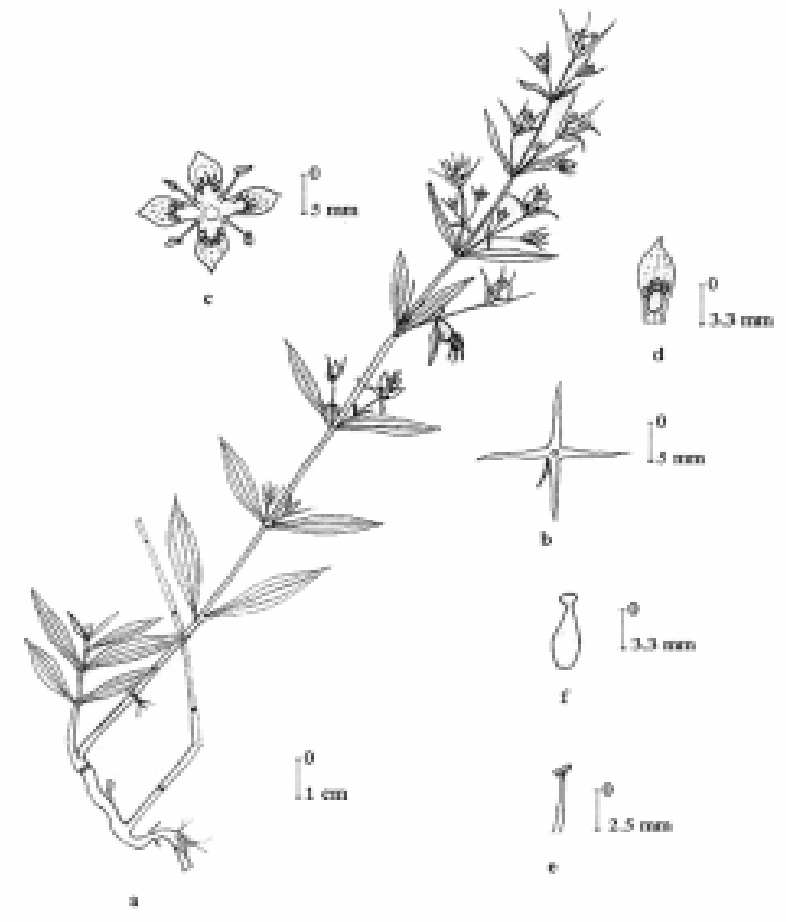

Fig. 1. Swertia angustifolia D. Don: (a) habit, (b) calyx, (c) corolla, (d) petal with nectary, (e) stamen, (f) carpel (Raskoti 10, TUCH).

2. Swertia chirayita (Roxb. ex Fleming) H. Karst., Deut. Fl. (Karsten): 1025 (1883). Clarke in Fl. Br. India: 4 (10): 124 (1883). Chater in Enum. Fl. Pl. Nepal 3: 96 (1982). Malla et al., Fl. Kath. Valley: 483 (1986). Aitken in Fl. Bhutan 2 (2): 626 (1999). Press et al., Ann. Check. Fl. Pl. Nepal: 118 (2000).

Swertia chirata (Wall.) C. B. Clarke in Fl. Br. India: 4: 124 (1883). Chiraito

Annual or biennial herb up to $90 \mathrm{~cm}$. Stem erect, hollow, terete, glabrous. Leaves sessile, leaf blade ovate or elliptic, 1.6-10.2 × 0.6$4.0 \mathrm{~cm}$, amplexicaul or clasping, margin entire, apex acute. Inflorescence panicles of cymes. Flowers 4-merous. Pedicels 0.4- 
$0.6 \mathrm{~cm}$. Bracts elliptic-ovate, $0.5-1.0 \mathrm{~cm} \times 1-3 \mathrm{~mm}$. Calyx greenish yellow, tube 0.5-1.0 mm, lobes linear lanceolate, 4-6 × 0.5-1.0 mm. Corolla greenish yellow, tube 1-2 mm, lobes ovate, 4-6.5 × 2-3 mm, glands 2 per corolla lobe, oval or oblong, fimbriate. Stamens 4 , haplostemonous, filament 3-4 mm, anthers 0.5-1.0 mm. Carpel 3-5 mm; stigma lobe capitate. Capsule 0.5-0.6 cm. Fl. \& Fr. Sept.-Nov. (Fig. 2).

Distribution: Nepal (CE, 1500-2500 m), Himalaya (Kashmir to Bhutan), NE India.

Specimens examined: Taplejung: Change-8, 2100 m, 13 Oct. 2002, P. Paudel 101d (TUCH); Ghunsa khola, 2500 m, 5 Sept. 1989, C. Grey-Wilson et al. 243 (KATH); Hurure-Mure, 31 Aug. 1986, T.B. Shreshtha and P.R. Shakya 8908 (KATH); Khapare, 25 Bhadra 2037, I. Shreshtha and T.M. Shrestha 33 (KATH). Dolakha: Dusikharka, 2400 m, 4 July 1990, N.K. Bhattarai 90/417 (KATH). Sindhupalchok: Kakani, 2000 m, 3 Nov. 1985, N.K. Bhattarai 85/827 (KATH). Kathmandu: Manichur, 1900 m, 15 Sept. 2000, G. Dongol 15 (TUCH); Nagarjun, 1676 m, 19 Oct. 1964, Bista et al. 9655 (KATH). Lalitpur: Phulchowki, 1750 m, 10 Aswin 2055, N. Thapa 45 (TUCH). Gorkha: S-facing, 2740 m, 2 Oct. 1995, Olsen 355 (KATH). Kaski: Dhampus, 1650 m, 21 May 1992, N.P. Manandhar and S.K. Acharya 28-92 (KATH). Jumla: Rara, 2900 m, 10 Aug. 1981, N.P. Manandhar and D.P. Joshi 8123 (KATH). Doti: Khaptad, 3200 m, 12 Sept. 1983, L.R. Sharma and Kurmi 57/11 (KATH).

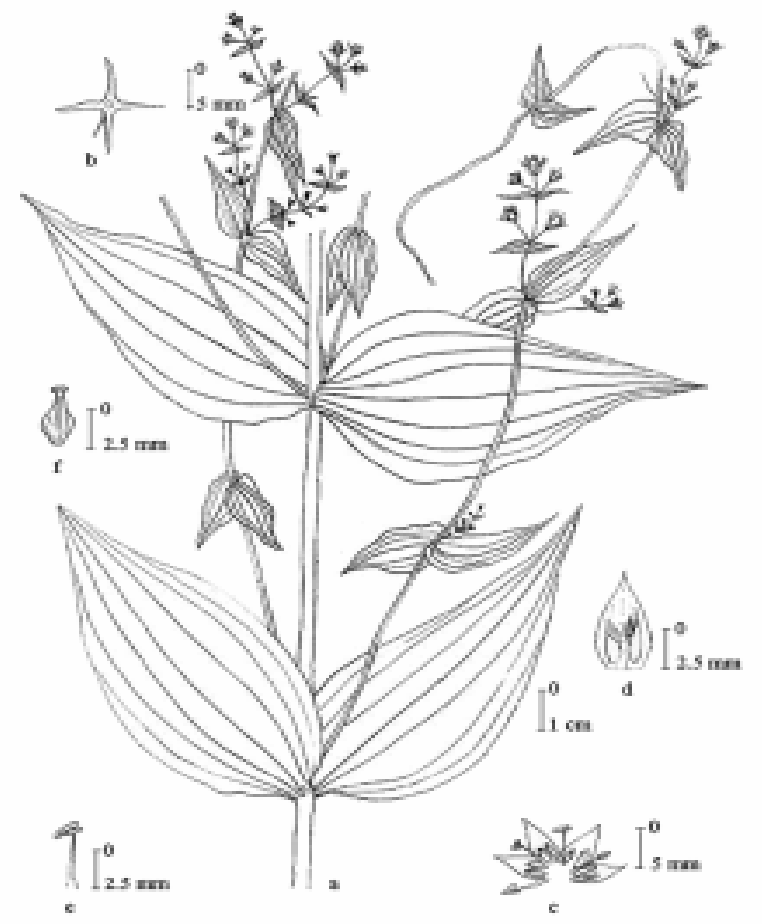

Fig 2. Swertia chirayita (Roxb. ex Fleming) H. Karst. (a) habit, (b) calyx, (c) corolla, (d) petal with nectarines, (e) stamen, (f) carpel (Paudel 101d, TUCH).

3. Swertia ciliata (D. Don ex G. Don) B.L. Burtt, Notes Roy. Bot. Gard. Edinburgh 26: 272 (1965). Chater in Enum. Fl. Pl. Nepal 3: 96 (1982). Malla et al., Fl. Kath. Valley: 483 (1986). Ting-nung and Pringle in Fl. China 16: 122 (1995). Aitken in Fl. Bhutan 2 (2): 623 (1999). Press et al., Ann. Check. Fl. Pl. Nepal: 118 (2000).

Ophelia ciliata D. Don ex G. Don, Gen. Syst. 4: 178 (1837).

Kalen
Annual herb up to $50 \mathrm{~cm}$. Stem erect, hollow, glabrous, branched. Leaves sessile or short petiolate, leaf blade lanceolate to ovate-lanceolate, 0.8-3.5 × 0.2-0.6 cm, base obtuse, margin slightly revolute, apex acute, veins 3. Inflorescence panicles of cymes. Flowers 5-merous. Pedicel 0.4-2.0 cm. Bracts lanceolate, 1-2 cm × 2-4 $\mathrm{mm}$. Calyx green, tube 1-2 mm, lobes reflexed at anthesis, lanceolate, 0.5-0.6 cm $\times 1.5-2.0 \mathrm{~mm}$, apex acuminate. Corolla pale blue or bluish white, with purple band above gland in each petal; tube 1.0-1.5 mm, lobes spreading at anthesis, ovate-lanceolate, $4 \times 2 \mathrm{~mm}$, apex acuminate. Nectary 1 per corolla lobe, horse-shoe-shaped, naked. Stamens 5, haplo-stemonous; filaments dark purple, 3.5-5.5 mm, connate at the base around ovary forming a rim or tube, tube 1.5-2.0 $\mathrm{mm}$, anthers blue purple, $1.0 \times 0.5 \mathrm{~mm}$. Carpel $1.5-5 \mathrm{~mm}$, style $1-2 \mathrm{~mm}$, stigmas capitate. Capsule 7-9 mm. Fl. \& Fr. Jul.-Sept. (Fig. 3).

Distribution: Nepal (WCE, 1700-4000 m), Afghanistan, Himalaya (Kashmir to Sikkim).

Specimens examined: East Nepal: between Basantpur and Doorpani, 2690 m, C. Grey-Wilson 50 (KATH). Central Nepal: Deurali-Chitre, 23002960 m, 17 Sept. 1985, N. Dabadi et al. 67/85 (KATH). Gorkha: Ghyalchok, 3600 m, 18 Sept. 1995, Olsen 337 (KATH). Manang: between Gunsang and Yak Kharka, 3900 m, Sept. 2005, D.P. Rijal 125M (TUCH). Jumla: 2800 m,

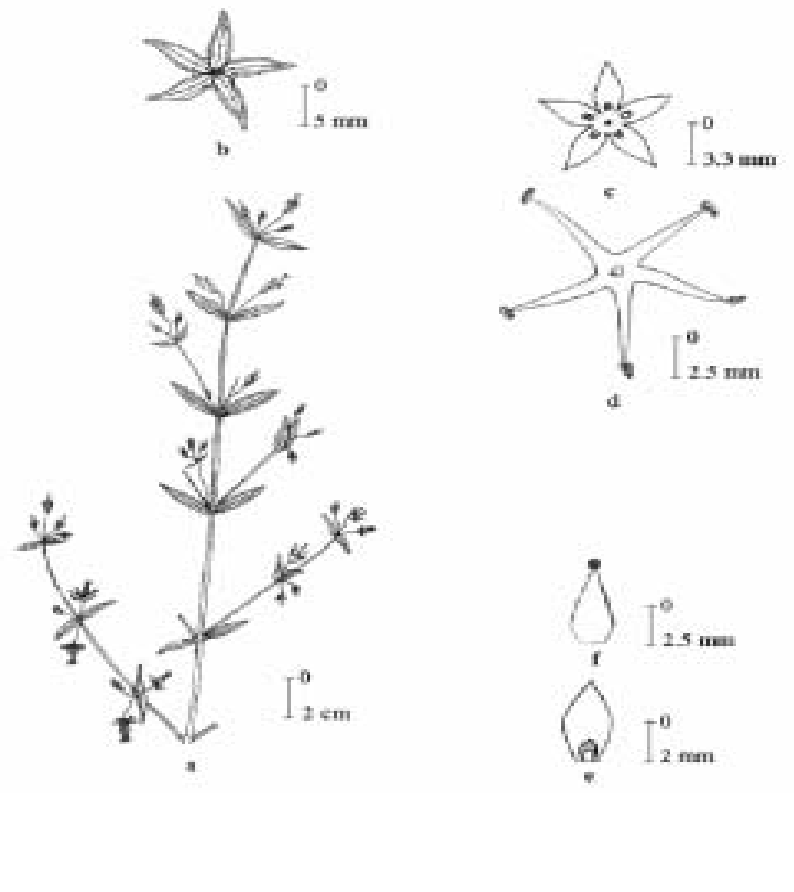

Fig. 3. Swertia ciliata (D. Don ex G. Don) B.L. Burtt. (a) habit, (b) calyx, (c) corolla, (d) androecium, (e) petal with nectary, (f) carpel (Rijal 125M, TUCH).

6 Sept. 1973, Einemon et al. 3871 (KATH). Doti: Khaptad, 3200 m, 2 Sept. 1984, L.R. Sharma and P.P. Kurmi 4295 (KATH).

4. Swertia cuneata D. Don, London Edinb. Philos. Mag. J. Sci. 8: 77 (1836). Clarke in Fl. Br. India: 4 (10): 129 (1883). Chater in Enum. Fl. Pl. Nepal 3: 96 (1982). Ting-nung and Pringle in Fl. China 16: 109 (1995). Aitken in Fl. Bhutan 2 (2): 628 (1999). Press et al., Ann. Check. Fl. Pl. Nepal: 118 (2000). 
Perennial herb 9-60 cm. Stem erect, hollow, glabrous; base sheathed by blackish remains of old petioles. Leaves mostly basal, whorled; petiole winged, up to $2.3 \mathrm{~cm}$, leaf blade spatulate-obovate, 1.0$5.0 \times 0.5-1.0 \mathrm{~cm}$, base attenuate, margin entire, apex rounded or acute. Stem leaves 2 or 3 pairs, sessile or short petiolate, petiole 1$2 \mathrm{~cm}$, leaf blade narrowly elliptic, 4.0-5.0 $\times 1.0 \mathrm{~cm}$, veins 3-7. Inflorescence panicles of cymes. Flowers 5-merous. Bracts linear or narrowly oblong, 2-3.5 × 0.2-0.7 cm. Pedicel 1.3-5.5 cm. Calyx tube 1.0-1.5 mm, lobes oblong-spatulate to elliptic, $4.5-10 \mathrm{~mm}$. Corolla blue or pale-blue or dull purple, tube $2.0 \mathrm{~mm}$, lobes narrowly elliptic or ovate, hairy at base, $1.7 \times 0.6 \mathrm{~cm}$, veins 5-7. Nectaries 2 per corolla lobe, linear, fimbriate, fimbriae up to $4 \mathrm{~mm}$. Stamens 5 , haplostemonous; filaments $0.9-1.0 \mathrm{~cm}$, anthers blue, $3 \mathrm{~mm}$, versatile. Carpel 1-2 cm, style indistinct, stigma bilobed. Capsule 1.8-2 cm. Fl. \& Fr. Aug.-Oct. (Fig. 4).

Distribution: Nepal (WCE, 3900-5000 m), Himalaya (Uttar Pradesh to Sikkim), NE India, China (Xizang).

Specimens examined: Rasuwa: Lawri Binayak, 4200 m, 26 Aug. 1969, Malla 16201 (KATH); Ghopte to Gosaithan, 4267 m, 9 Sept. 1965, T.B. Shrestha 3741 (KATH). Manang: Chhonkar, 4300 m, Sept. 2005, D.P. Rijal 150M (TUCH). Jangla Bhanjyang, 4267 m, 15 Aug. 1973, Einemon et al. 3242 (KATH).
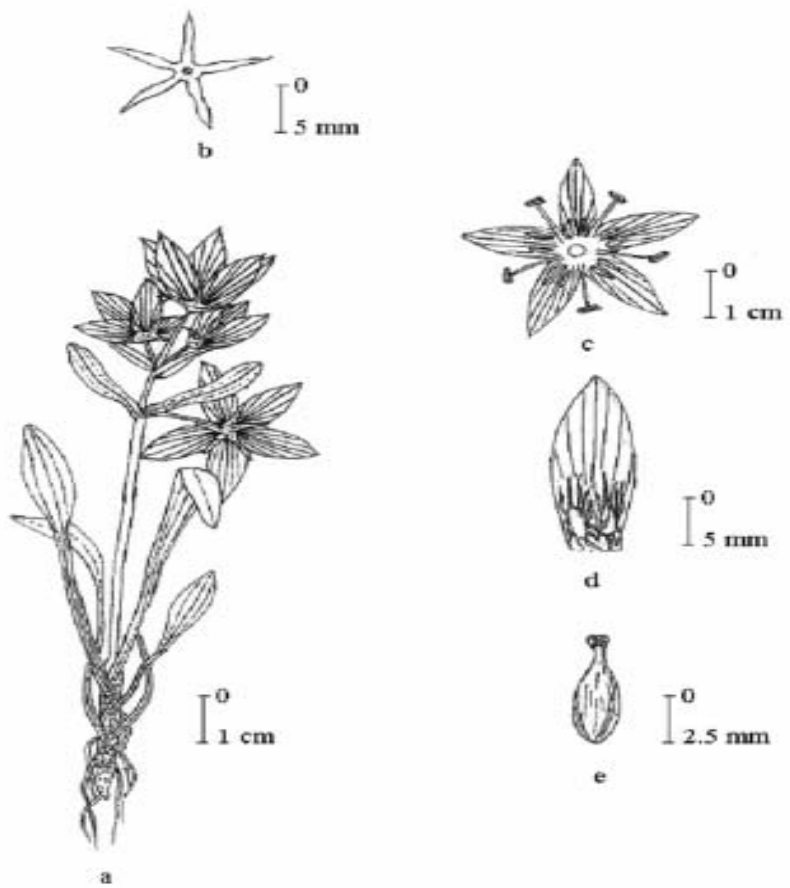

Fig. 4. Swertia cuneata D. Don (a) habit, (b) calyx, (c) corolla and androecium, (d) petal with nectarines, (e) carpel (Rijal 150M, TUCH).

5. Swertia dilatata var. pilosa C.B. Clarke, Fl. Br. India: 4(10): 122 (1883). Chater in Enum. Fl. Pl. Nepal 3: 96 (1982). Malla et al., Fl. Kath. Valley: 484 (1986). Press et al., Ann. Check. Fl. Pl. Nepal: 118 (2000).

Swertia dilatata Malla et al., Fl. Kath. Valley: 484 (1986).

Herb up to $50 \mathrm{~cm}$. Stem erect, hollow, terete at base and cylindrical above, glabrous. Leaves sessile, leaf blade linear or narrowly elliptic-lanceolate, recurved downwards, $1.8-4.0 \mathrm{~cm} \times 2-4$ mm, base minutely ciliate and attenuate, margin entire, apex acute. Inflorescence panicles of cymes. Flowers 5-merous. Pedicel 0.3$1.5 \mathrm{~cm}$.. Bracts elliptic lanceolate, 1.6-3.0 × 0.2-0.4 cm. Calyx green, tube 1.0-1.5 mm, lobe ovate or lanceolate, 6.5-7.5 $\times$ 4.0-5.0 mm. Nectary 1 in each corolla lobe, horse-shoe-shaped, flap without fimbria. Stamens 5, haplostemonous, filament much dilated for more than half their length, $4-5 \mathrm{~mm}$, anthers $1.0 \times 0.5 \mathrm{~mm}$. Capsule $8 \times 2.5$ mm. Fl. \& Fr. Sept.-Nov. (Fig. 5).

Distribution: Nepal (WCE, 1400-4100 m).

Specimens examined: East Nepal: Murhay-Chitre, 24 Oct. 1963, H. Hara et al. 6302545 (KATH). Phidim: Inthung, 2400 m, 19 Nov. 1978, P. Pradhan et al. 1071 (KATH). Sankhuwasabha: Chauki, 2690 m, 12 Oct. 2002, P. Paudel 101e (TUCH). Kathmandu: Manichur, 2030 m, 6 Sept. 2000, S. Marasani 46 (TUCH). Myagdi: Tadopani, 2700 m, Oct. 1999, Sita and Puspa sp169 (TUCH). Baglung: Lephane, 2300 m, 11 Aswin 2056, P. Sapkota 5 (TUCH). Jumla: 4115 m, 23 Sept. 1983, N.P. Manandhar 9042 (KATH). Surkhet: Goganpani, 1460 m, 8 Dec. 1982, S.R. Adhikari and N.P. Manandhar 9198 (KATH). Bajhang: Jhogun danda, 2250 m, 27 Aug. 1972, M.S. Bista and D.P. Joshi 757 (KATH).

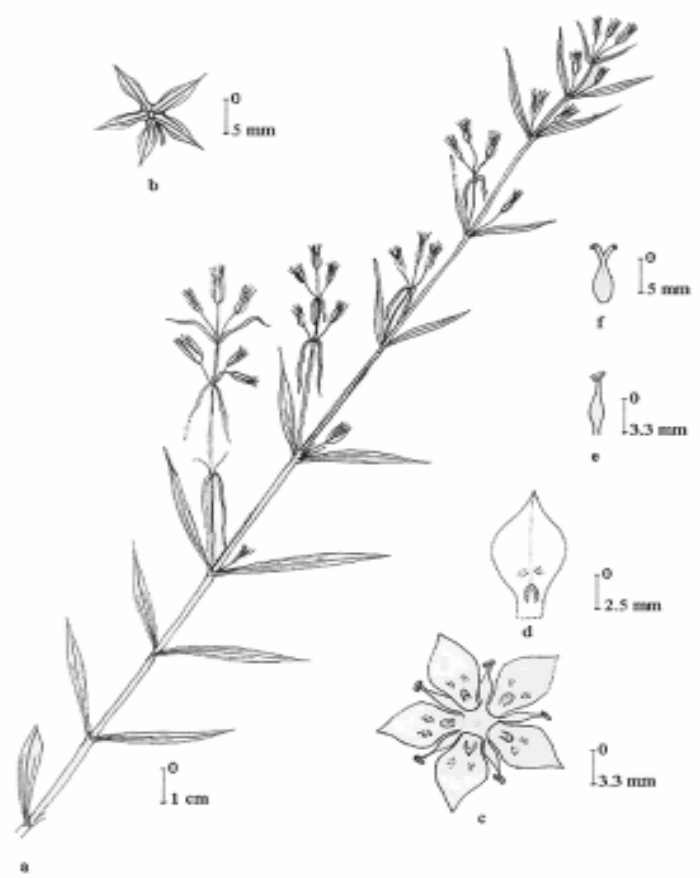

Fig. 5. Swertia dilatata var. pilosa C.B. Clarke (a) habit, (b) calyx, (c) corolla, (d) petal with nectary, (e) stamen, (f) capsule (Marasani 46, TUCH).

6. Swertia nervosa (G. Don) C. B. Clarke, Fl. Br. India: 4 (10): 125 (1883). Chater in Enum. Fl. Pl. Nepal 3: 97 (1982). Malla et al., Fl. Kath. Valley: 484 (1986). Ting-nung and Pringle in Fl. China 16: 116 (1995). Aitken in Fl. Bhutan 2 (2): 626 (1999). Press et al., Ann. Check. Fl. Pl. Nepal: 118 (2000).

Ophelia nervosa (G. Don) Griseb., Gen. Sp. Syst. 317 (1838).

Kalochiraito 
Annual herb up to $100 \mathrm{~cm}$. Stem erect, hollow, sub-quadrangular, glabrous, winged. Leaves sessile or sub-sessile, leaf blade elliptic to lanceolate, $2.4-7.1 \times 0.4-1.8 \mathrm{~cm}$, base attenuate, margin entire, apex acute, veins 1-3. Inflorescence panicles of cymes. Pedicel 0.5$2.0 \mathrm{~cm}$. Bracts linear or lanceolate, 1.3-2.5 cm × 2-4 mm. Flowers 4merous. Calyx green, tube 0.5-1.0 mm, lobes linear-lanceolate, 1.0$1.3 \mathrm{~cm} \times 1-2 \mathrm{~mm}$. Corolla pale yellow-green or whitish, with purple veins, tube1-2 mm, lobes elliptic-ovate, $0.7-0.8 \mathrm{~cm} \times 2-3 \mathrm{~mm}$. Nectary 1 per corolla lobe, with pocket-like flap, fimbriate at apex. Stamens 4, haplostemonous, filament 4.5-6.0 mm; anthers $1 \mathrm{~mm}$. Carpel 0.7$0.8 \mathrm{~cm}$; ovary ovoid, style short, stigma lobe capitate. Capsule 0.5 cm. Fl. \& Fr. Aug.-Nov. (Fig. 6).

Distribution: Nepal (WCE, 700-3200 m), Himalaya (Himanchal Pradesh to Bhutan), NE India (Assam, Nagaland), W. China.

Specimens examined: Terhathum: Shreemane, 2690 m, 12 Oct. 2002, P. Paudel 101d (TUCH). Sindhupalchowk: south of Ganja pass, 4000 m, 26 Sept. 1966, D.H. Nicolson 2647 (KATH). Katmandu: Sundarijal, 16 Feb. 2001, B. Adhikari 152 (TUCH). Lalitpur: Phulchowki, 1600 m, 16 Shrawan 2056, M. Thapa (TUCH). Baglung: Dhapharkhet, 2300 m, 8 Sept. 1991, N.P. Manandhar 944-91 (KATH). Mustang: Muktinath, 3453 m, 23 Sept. 1985, D.P. Joshi and N.K. Bhattarai 166/85 (KATH). Mugu: Gamgarhi, 3200 m, 10 Aug. 1981, N.P. Manandhar and D.P. Joshi 6981 (KATH); Rara National Park, 3200 m, 26 Nov. 1995, P.P. Kurmi and N. Thapa 7468 (KATH). Jajarkot: Samaila, 2130 m, 17 Sept. 1982, N.P. Manandhar 8955 (KATH).

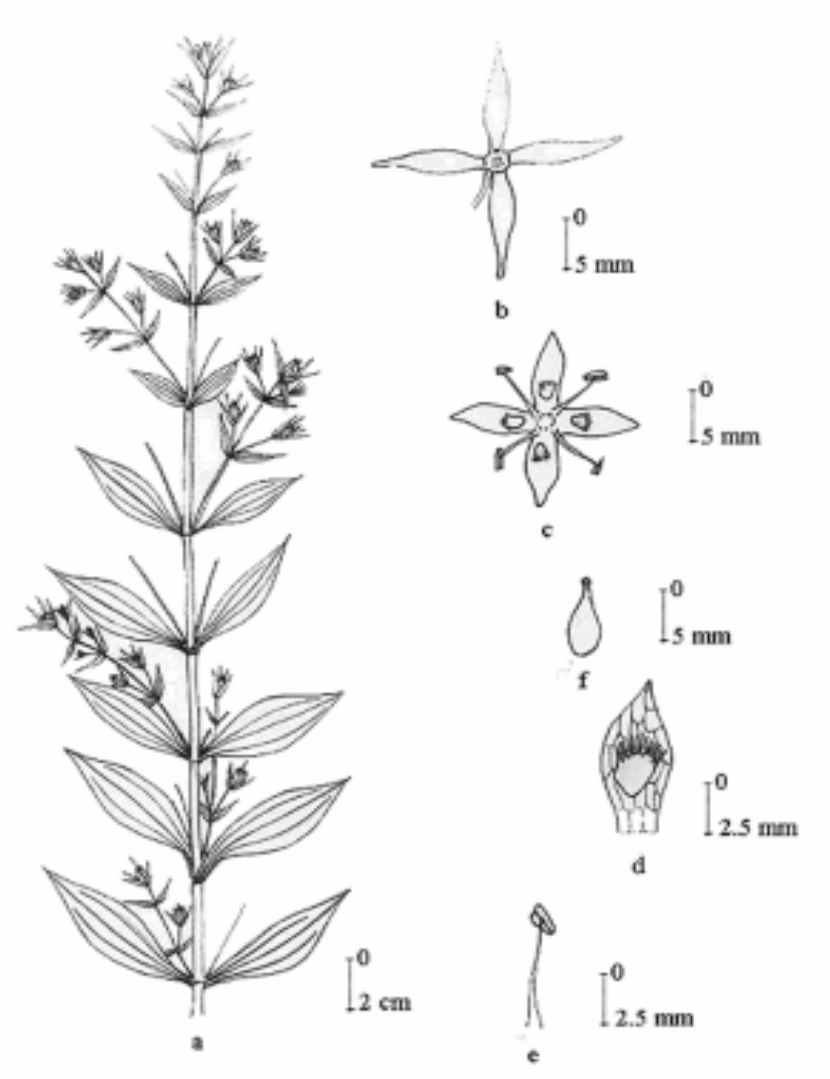

Fig. 6. Swertia nervosa (G. Don) C. B. Clarke (a) habit, (b) calyx, (c) corolla, (d) petal with nectary, (e) stamen, (f) carpel (Paudel 101d, TUCH).
7. Swertia paniculata Wall., Pl. Asiat. Rar. 3 (9): 3, t. 205 (1831). Clarke in Fl. Br. India: 4(10): 122 (1883). Chater in Enum. Fl. Pl. Nepal 3: 97 (1982). Ting-nung and Pringle in Fl. China 16: 123 (1995). Aitken in Fl. Bhutan 2 (2): 624 (1999). Press et al., Ann. Check. Fl. Pl. Nepal: 118 (2000).

Ophelia paniculata (Wall.) D. Don in Tr. Linn. S. 17: 525 (1837).

Annual herb, 80-120 cm. Stem erect, hollow, terete, glabrous. Leaves sessile, blade linear, $1.0-2.5 \mathrm{~cm} \times 1-3 \mathrm{~mm}$, base attenuate, margin entire, apex acuminate, veins $1-3$. Inflorescence axillary and terminal, panicles of cymes. Pedicels $0.3-1.0 \mathrm{~cm}$. Bracts linear lanceolate, $0.5-1.1 \mathrm{~cm} \times 0.5-1.0 \mathrm{~mm}$. Calyx tube $0.5 \mathrm{~mm}$, lobes lanceolate, $4 \times 1 \mathrm{~mm}$, 3-nerved. Corolla pale yellow; tube1 mm, lobes elliptic-ovate, $5 \times 2.5 \mathrm{~mm}$, twisted. Nectary 1 per corolla lobe, horse-shoe-shaped, opening towards base, non-fimbriated yellowish flap. Stamens 5, haplostemonous, filament $2.5 \mathrm{~mm}$, anther $1 \mathrm{~mm}$. Carpel $4 \times 1 \mathrm{~mm}$. Fl. \& Fr. Aug.-Sept. (Fig. 7).

Distribution: Nepal (WCE, 1500-4000 m), Himalaya (Kashmir to Bhutan), NE India, Myanmar, China (Xizang).

Specimens examined: Way to Tatopani, 2395 m, 28 Sept. 1994, Y. Piya 60 (TUCH). Khapare, 25 Bhadra 2037, I. Shreshtha and T.H. Shrestha 14 (KATH). Makawanpur: Shimbhanjyang, 2438 m, Oct. 1960, S.B. Shah 17 (KATH). Sangasoti Bangtholi, 2316 m, 8 Oct 1960, S.B. Malla and S.B. Rajbhandary 349 (KATH). Mustang: Lete-Larjung Cobang, 2280-2480 m, 20 Sept. 1985, N. Dabadi and N. Pradhan 134a (KATH). Jharkot, 3300 m, 17 Aswin 2051, M.K. Bhandari 48 (TUCH).

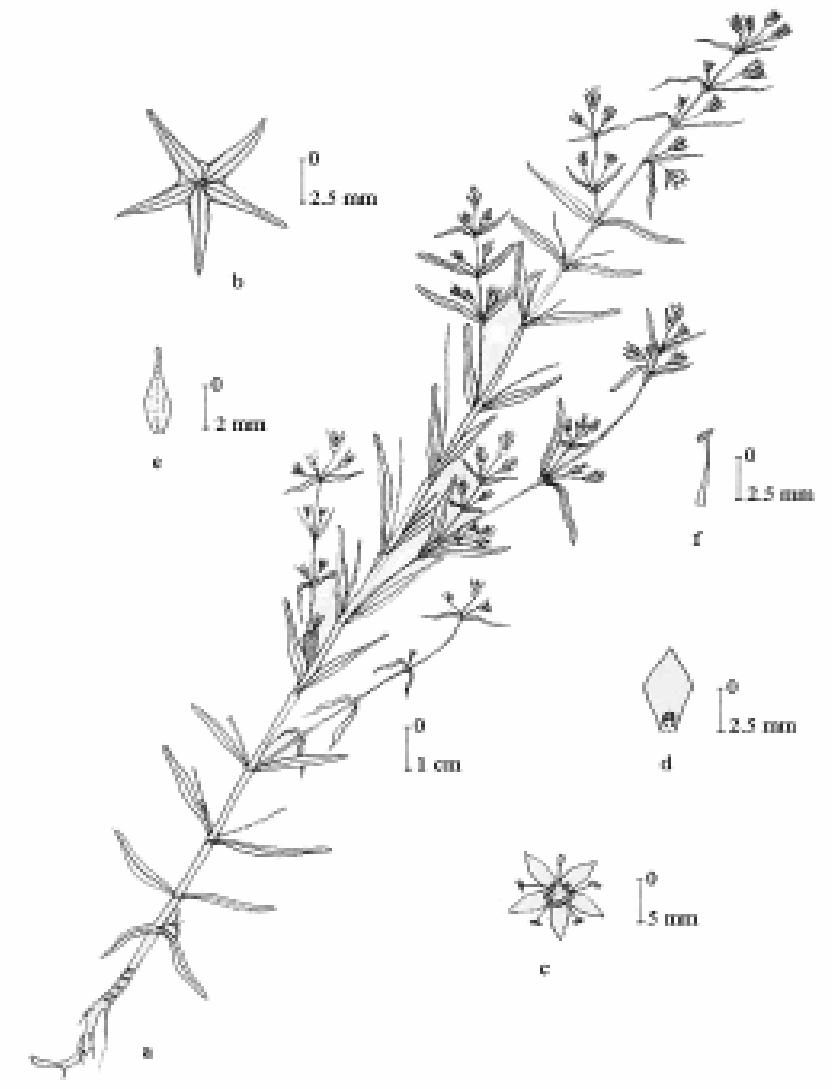

Fig. 7. Swertia paniculata Wall. (a) habit, (b) calyx, (c) corolla, (d) petal with nectary, (e) carpel, (f) stamen (Bhandari 48, TUCH). 
8. Swertia racemosa (Griseb.) C. B. Clarke, Fl. Br. India: 4 (10): 124 (1883). Chater in Enum. Fl. Pl. Nepal 3: 97 (1982). Ting-nung and Pringle in Fl. China 16: 123 (1995). Aitken in Fl. Bhutan 2 (2): 625(1999). Press et al., Ann. Check. Fl. Pl. Nepal: 118 (2000).

Ophelia racemosa Griseb., Gen. Sp. Gent. 319 (1838).

Herb, 3-40 cm. Stem erect, hollow, terete, glabrous. Leaves sessile, blade lanceolate, $1.5-4.0 \times 0.6-1.2 \mathrm{~cm}$, base auriculate and sub-amplexicaul, margin entire, ciliate, apex acute, veins 1-3. Inflorescence panicles of cymes, many flowered, spreading. Pedicel 0.8-2.0 cm. Flowers 5-merous. Calyx green, tube campanulate, 3-4 $\mathrm{mm}$, lobes triangular lanceolate, margin ciliate, 3.5-5 mm. Corolla pale blue to pale blue-purple, tube campanulate, $9 \mathrm{~mm}$, lobe 3-6 mm, margin ciliate. Nectary 1 in each petal, linear, fimbriate, opening towards the base. Stamens 5, haplostemonous, filament basally white, apically blue, 5-7 mm, basally much enlarged and connate, anthers blue. Capsule 0.7-1.5 cm. Fl. \& Fr. Aug.-Sept. (Fig. 8).

Distribution: Nepal (WCE, 1700-5000 m), Himalaya (Nepal to Bhutan), NE India, China (Xizang).

Specimens examined: Sindhupalchowk: south of Ganja pass, $4000 \mathrm{~m}$, 26 Sept. 1966, D.H. Nicolson 2647 (KATH). Kathmandu: Sundarijal, 1700 m, 1 Jestha 2057, S. Gautam 53 (TUCH). Rasuwa: Langtang, April 1999, A. Pokhrel and G. Rana AG248 (TUCH). Manang: Way to Tilicho, $4000 \mathrm{~m}$, Sept. 2005, D.P. Rijal 115M (TUCH). Mustang: Muktinath, 3795 m, 25 Sept. 2003, B. Dewan 144 (TUCH). Baglung: Jaljala to Gurjaghat, 34803018 m, 10 June 1985, D.P. Joshi et al. 212/85 (KATH). Dhorpatan, 2896 m, 16 Oct. 1954, Stainton et al. 9056 (KATH). Mugu: Rara, 10 Aug. 1981, N. Dabadi and N. Pradhan 8084 (KATH).

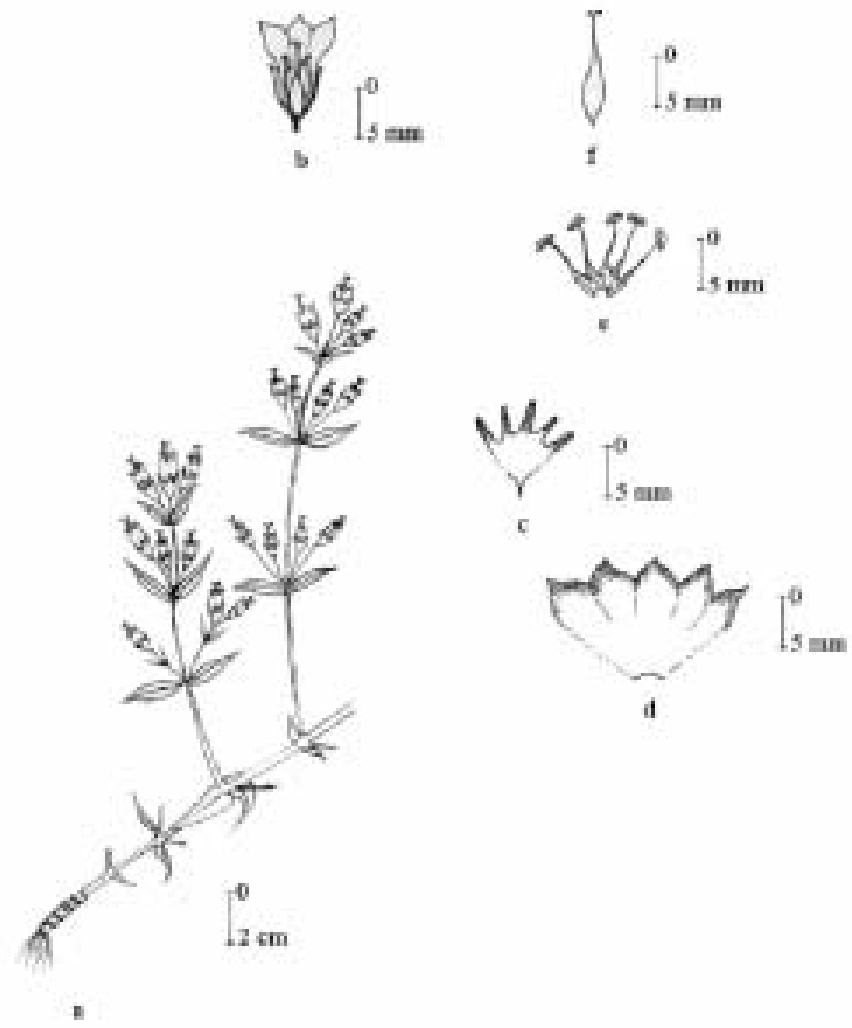

Fig. 8. Swertia racemosa (Griseb.) C.B. Clarke (a) habit, (b) l.s. of flower, (c) calyx, (d) corolla, (e) stamen, (f) carpel (Gautam 53, TUCH).

\section{Discussion}

The present study was based on only eight species of Swertia ( $S$. angustifolia, S. chirayita, S. ciliata, S. cuneata, S. dilatata, S. nervosa, S. paniculata, and S. racemosa) out of a total of 30 species so far recorded from Nepal. All the speceis included in this study are distributed from west to east Nepal. Lack of sufficient herbarium specimen was the main factor behind the study of only eight species of Swertia. However, important and confusing species, which are commonly adulterated with $S$. chirayita in trade, have been included. This study has identified some important morphological characters to distinguish these species. The present study also provides the basic information for the detailed study of the genus Swertia in future.

Petal color, number of floral parts (tetramerous vs. pentamerous), number of nectaries (glands) in petal (1 or 2), characteristics of flap (presence or absence and either fimbriated or non-fimbriated), and presence or absence of cilia are the key characters for the delimitation of the species of Swertia. These characteristics must be considered for correct identification of the species commonly adulterated with $S$. chirayita. Furthermore, the presence of cilia or papillae is quite prominent in S. ciliata and not distinct in all other species. Out of the three tetramerous species studied, only $S$. chirayita contains two glands. Among the pentamerous species, $S$. cuneata contains two glands. S. cuneata and S. racemosa contain fimbriated flap on the gland. Presence of two green spots above each gland is prominent only in S. dilatata. The later species is further differentiated from all other species based on the presence of distinctly dilated filaments.

\section{Acknowledgements}

I am very grateful to Prof. Dr. Ram P. Chaudhary, Prof. Dr. Krishna K. Shrestha, Ms. Sangeeta Rajbhandary and Mr. Bhaskar Adhikari for their constant support, suggestions, and encouragement during this study. I would like to express my sincere gratitude to Prof. Dr. Pramod Kumar Jha, former Head of the Central Department of Botany, Tribhuvan University for providing necessary laboratory facilities. I would like to thank the curators of TUCH and KATH for providing necessary specimens for the study.

\section{References}

Bhattarai K.R. and Acharya N. 1998. Swertia species (chiraito) of commerce in Nepal. Plant Research 1(1): 48-55.

Chassot P. 2003. A new species of Swertia L. (Gentianaceae) from Nepal. Botanical Journal of Linnean Society 141: 389-394.

Clarke C.B. 1985. Gentianaceae. In: Flora of British India, Vol. IV (J.D. Hooker, ed.), pp. 121-130. L. Reeve and Co. Ltd., Ashford, Kent, UK. Cronquist A. 1988. The Evolution and Classification of Flowering Plants. The New York Botanical Garden, Bronx, New York, USA.

DPR 2001. Flowering Plants of Nepal (Phanerogams). His Majesty's Government, Ministry of Forest and Soil Conservation. Department of 
Plant Resources, Kathmandu, Nepal.

Ghimire S.K., Pyakurel D., Nepal B., Sapkota I.B., Parajuli R.R. and Oli B.R. 2008. Gair Kasta Banpaidawar Digdarshan (manual of NTFPs of Nepal Himalaya). WWF Nepal, Kathmandu, Nepal (in Nepali).

Grierson A.J.C. and Long D.G. 1999. Flora of Bhutan Vol 2 part 2. Royal Botanic Garden, Edinburgh, UK.

Judd W.S., Campbell C.S., Kellog E.A. and Stevens P.F. 1999. Plant Systematics

- A Phylogenetic Approach. Sinauer Associates Inc., Sunderland,
Massachusetts, USA.

Pant S.R. and Bimb H.P. 2005. Morphological variation in Swertia nervosa (G. Don) C. B. Clarke of Nepal. Nepal Journal of Plant Sciences 1: 7882.

Press J.R., Shrestha K.K. and Sutton D.A. 2000. Annotated Checklist of the Flowering Plants of Nepal. The Natural History Museum, London, UK.

Zheng-Yi W. and Raven P.H. 1995. Flora of China, Vol. 16. Missouri Botanical Garden, St. Louis, USA and Chinese Academy of Sciences, Beijing, China. 